\title{
Subclass of Multivalent Harmonic Functions Defined by Wright Generalized Hypergeometric Functions
}

\author{
M. K. Aouf, A. O. Moustafa, and E. A. Adwan \\ Department of Mathematics, Faculty of Science, Mansoura University, Mansoura 35516, Egypt \\ Correspondence should be addressed to E. A. Adwan; eman.a2009@yahoo.com \\ Received 13 May 2013; Revised 10 September 2013; Accepted 10 September 2013 \\ Academic Editor: J. Dziok
}

Copyright ( 2013 M. K. Aouf et al. This is an open access article distributed under the Creative Commons Attribution License, which permits unrestricted use, distribution, and reproduction in any medium, provided the original work is properly cited.

\begin{abstract}
We introduce a new class of multivalent harmonic functions defined by Wright generalized hypergeometric function. Coefficient
\end{abstract} estimates, extreme points, distortion bounds, and convex combination for functions belonging to this class are obtained.

\section{Introduction}

A continuous complex-valued function $f=u+i v$ defined in a simply connected complex domain $D$ is said to be harmonic in $D$ if both $u$ and $v$ are real harmonic in $D$. In any simply connected domain the function $f(z)$ can be written in the form

$$
f=h+\bar{g},
$$

where $h$ and $g$ are analytic in $D, h$ is called the analytic part, and $g$ the coanalytic part of $f$. A necessary and sufficient condition for $f$ to be locally univalent and sense-preserving in $D$ is that $\left|h^{\prime}(z)\right|>\left|g^{\prime}(z)\right|$ in $D$ (see [1]).

Denote by $S_{H}$ the class of functions $f$ of the form (1) that are harmonic univalent and sense preserving in the unit disc $U=\{z:|z|<1\}$ for which $f(0)=f_{z}(0)-1=0$.

Recently, Ahuja and Jahangiri [2] defined the class $H_{p}(p \in \mathbb{N}=\{1,2, \ldots\})$ consisting of all $p$-valent harmonic functions $f=h+\bar{g}$ that are sense-preserving in $U$ and $h, g$ are of the form

$$
h(z)=z^{p}+\sum_{n=1+p}^{\infty} a_{n} z^{n}, \quad g(z)=\sum_{n=p}^{\infty} b_{n} z^{n}, \quad\left|b_{p}\right|<1 .
$$

Let $\alpha_{1}, A_{1}, \ldots, \alpha_{q}, A_{q}$ and $\beta_{1}, B_{1}, \ldots, \beta_{s}, B_{s}\left(q, s \in \mathbb{N}_{0}=\right.$ $\mathbb{N} \cup\{0\}$ ) be positive real parameters such that

$$
1+\sum_{j=1}^{s} B_{j}-\sum_{j=1}^{q} A_{j} \geq 0
$$

The Wright generalized hypergeometric function [3] (see also [4])

$$
\begin{aligned}
{ }_{q} \Psi_{s} & {\left[\left(\alpha_{1}, A_{1}\right), \ldots,\left(\alpha_{q}, A_{q}\right) ;\left(\beta_{1}, B_{1}\right), \ldots,\left(\beta_{s}, B_{s}\right) ; z\right] } \\
& ={ }_{q} \Psi_{s}\left[\left(\alpha_{i}, A_{i}\right)_{1, q} ;\left(\beta_{i}, B_{i}\right)_{1, s} ; z\right]
\end{aligned}
$$

is defined by

$$
\begin{aligned}
q_{q} \Psi_{s} & {\left[\left(\alpha_{i}, A_{i}\right)_{1, q} ;\left(\beta_{i}, B_{i}\right)_{1, s} ; z\right] } \\
& =\sum_{n=0}^{\infty} \frac{\prod_{i=1}^{q} \Gamma\left(\alpha_{i}+n A_{i}\right)}{\prod_{i=1}^{s} \Gamma\left(\beta_{i}+n B_{i}\right)} \cdot \frac{z^{n}}{n !} \quad(z \in U) .
\end{aligned}
$$

If $A_{i}=1(i=1, \ldots, q)$ and $B_{i}=1(i=1, \ldots, s)$, we have the relationship

$$
\Omega_{q} \Psi_{s}\left[\left(\alpha_{i}, 1\right)_{1, q} ;\left(\beta_{i}, 1\right)_{1, s} ; z\right]={ }_{q} F_{s}\left(\alpha_{1}, \ldots, \alpha_{q} ; \beta_{1}, \ldots, \beta_{s} ; z\right),
$$

where ${ }_{q} F_{s}\left(\alpha_{1}, \ldots, \alpha_{q} ; \beta_{1}, \ldots, \beta_{s} ; z\right)$ is the generalized hypergeometric function (see [4]) and

$$
\Omega=\frac{\prod_{i=1}^{s} \Gamma\left(\beta_{i}\right)}{\prod_{i=1}^{q} \Gamma\left(\alpha_{i}\right)} .
$$

By using the generalized hypergeometric function, Dziok and Srivastava [5] introduced a linear operator. Dziok and Raina in [6] and Aouf and Dziok in [7] extended this linear operator by using Wright generalized hypergeometric function. 
Aouf et al. [8] defined the linear operator $\theta_{p, q, s}\left[\left(\alpha_{i}, A_{i}\right)_{1, q}\right.$; $\left.\left(\beta_{i}, B_{i}\right)_{1, s}\right]$ by the Hadamard product as

$$
\begin{aligned}
\theta_{p, q, s} & {\left[\left(\alpha_{i}, A_{i}\right)_{1, q} ;\left(\beta_{i}, B_{i}\right)_{1, s}\right] f(z) } \\
& ={ }_{q} \Phi_{s}^{p}\left[\left(\alpha_{i}, A_{i}\right)_{1, q} ;\left(\beta_{i}, B_{i}\right)_{1, s} ; z\right] * \varphi(z),
\end{aligned}
$$

where ${ }_{q} \Phi_{s}^{p}\left[\left(\alpha_{i}, A_{i}\right)_{1, q} ;\left(\beta_{i}, B_{i}\right)_{1, s} ; z\right]$ is given by

$$
\begin{aligned}
{ }_{q} \Phi_{s}^{p} & {\left[\left(\alpha_{i}, A_{i}\right)_{1, q} ;\left(\beta_{i}, B_{i}\right)_{1, s} ; z\right] } \\
& =\Omega z_{q}^{p} \Psi_{s}\left[\left(\alpha_{i}, A_{i}\right)_{1, q} ;\left(\beta_{i}, B_{i}\right)_{1, s} ; z\right] .
\end{aligned}
$$

We observe that, for a function $\varphi(z)$ of the form (2), we have

$$
\begin{aligned}
\theta_{p, q, s} & {\left[\left(\alpha_{i}, A_{i}\right)_{1, q} ;\left(\beta_{i}, B_{i}\right)_{1, s}\right] \varphi(z) } \\
= & z^{p}+\sum_{n=1+p}^{\infty} \Omega \sigma_{n, p}\left(\alpha_{1}\right) a_{n} z^{n},
\end{aligned}
$$

where $\Omega$ is given by (7) and $\sigma_{n, p}\left(\alpha_{1}\right)$ is defined by

$$
\begin{aligned}
& \sigma_{n, p}\left(\alpha_{1}\right) \\
& =\frac{\Gamma\left(\alpha_{1}+A_{1}(n-p)\right) \cdots \Gamma\left(\alpha_{q}+A_{q}(n-p)\right)}{\Gamma\left(\beta_{1}+B_{1}(n-p)\right) \cdots \Gamma\left(\beta_{s}+B_{s}(n-p)\right)(n-p) !} .
\end{aligned}
$$

For convenience, we write

$$
\begin{aligned}
\theta_{p, q, s} & {\left[\alpha_{1}, A_{1}, B_{1}\right] f(z) } \\
= & \theta_{p, q, s}\left[\left(\alpha_{1}, A_{1}\right), \ldots,\left(\alpha_{q}, A_{q}\right) ;\left(\beta_{1}, B_{1}\right), \ldots,\left(\beta_{s}, B_{s}\right)\right] \\
& \times \varphi(z) .
\end{aligned}
$$

Now we can define the modified Wright operator as follows:

$$
\begin{aligned}
\theta_{p, q, s}\left[\alpha_{1}, A_{1}, B_{1}\right] f(z)= & \theta_{p, q, s}\left[\alpha_{1}, A_{1}, B_{1}\right] h(z) \\
& +\overline{\theta_{p, q, s}\left[\alpha_{1}, A_{1}, B_{1}\right] g(z)}
\end{aligned}
$$

where

$$
\begin{gathered}
\theta_{p, q, s}\left[\alpha_{1}, A_{1}, B_{1}\right] h(z)=z^{p}+\sum_{n=1+p}^{\infty} \Omega \sigma_{n, p}\left(\alpha_{1}\right) a_{n} z^{n}, \\
\theta_{p, q, s}\left[\alpha_{1}, A_{1}, B_{1}\right] g(z)=\sum_{n=p}^{\infty} \Omega \sigma_{n, p}\left(\alpha_{1}\right) b_{n} z^{n}, \quad\left|b_{p}\right|<1 .
\end{gathered}
$$

For $p=1, \theta_{1, q, s}\left[\alpha_{1}, A_{1}, B_{1}\right] f(z)=W_{q}^{s}\left[\alpha_{1}\right] f(z)$, where $W_{q}^{s}\left[\alpha_{1}\right] f(z)$ is the modified Wright generalized hypergeometric functions (see [9]).

We note that, for $A_{i}=1(i=1,2, \ldots, q)$ and $B_{i}=1(i=$ $1,2, \ldots, s)$, we obtain $\theta_{p, q, s}\left[\alpha_{1}, 1,1\right] f(z)=H_{p, q, s}\left[\alpha_{1}\right] f(z)$, where $H_{p, q, s}\left[\alpha_{1}\right]$ is the modified Dziok-Srivastava operator (see [10]).

For $0 \leq \gamma<1, p \in \mathbb{N}$ and for all $z \in U$, let $H_{p}\left(\left[\alpha_{1}, A_{1}, B_{1}\right], q, s ; \gamma\right)$ denote the family of harmonic $p$ valent functions $f(z)=h(z)+\overline{g(z)}$, where $h$ and $g$ are given by (2) and satisfying the analytic criterion

$$
\begin{aligned}
\operatorname{Re}\left\{\left(z\left(\theta_{p, q, s}\left[\alpha_{1}, A_{1}, B_{1}\right] h(z)\right)^{\prime}\right.\right. \\
\left.\quad \overline{-z\left(\theta_{p, q, s}\left[\alpha_{1}, A_{1}, B_{1}\right] g(z)\right)^{\prime}}\right) \\
\times\left(\theta_{p, q, s}\left[\alpha_{1}, A_{1}, B_{1}\right] h(z)\right. \\
\left.\left.+\overline{\theta_{p, q, s}\left[\alpha_{1}, A_{1}, B_{1}\right] g(z)}\right)^{-1}\right\} \geq p \gamma .
\end{aligned}
$$

Let $\overline{H_{p}}\left(\left[\alpha_{1}, A_{1}, B_{1}\right], q, s ; \gamma\right)$ be the subclass of $H_{p}\left(\left[\alpha_{1}\right.\right.$, $\left.\left.A_{1}, B_{1}\right], q, s ; \gamma\right)$ consisting of functions $f=h+\bar{g}$ such that $h$ and $g$ are of the form

$$
h(z)=z^{p}-\sum_{n=1+p}^{\infty} a_{n} z^{n}, \quad g(z)=\sum_{n=p}^{\infty} b_{n} z^{n}, \quad\left|b_{p}\right|<1 .
$$

We note that for suitable choices of $q$ and $s$, we obtain the following subclasses:

(1) $\overline{H_{p}}([1,1,1], 2,1 ; \gamma)=S_{H}^{*}(p, \gamma)($ see $[2])$;

(2) $\overline{H_{p}}\left(\left[\alpha_{1}, 1,1\right], q, s ; \gamma\right)=T_{H}^{*}\left(p, \alpha_{1}, \gamma\right)($ see $[10])$;

(3) $\overline{H_{1}}\left(\left[\alpha_{1}, A_{1}, B_{1}\right], q, s ; \gamma\right)=W_{\bar{H}}\left(\left[\alpha_{1}\right], \gamma\right)$ (see $\left.[9]\right)$;

(4) $\overline{H_{1}}\left(\left[\alpha_{1}, 1,1\right], q, s ; \gamma\right)=V_{\bar{H}}\left(\alpha_{1}, \gamma\right)($ see $[11,12])$;

(5) $\overline{H_{1}}([1,1,1], 2,1 ; \gamma)=S_{H}^{*}(\gamma)($ see $[13])$.

\section{Coefficient Estimates}

Unless otherwise mentioned, we will assume in the reminder of this paper that the parameters $\alpha_{1}, A_{1}, \ldots, \alpha_{q}, A_{q}$ and $\beta_{1}, B_{1}, \ldots, \beta_{s}, B_{s}(q, s \in \mathbb{N})$ are positive real numbers, $0 \leq \gamma<$ $1, p \in \mathbb{N}, z \in U, \Omega$ is defined by (7), and $\sigma_{n, p}\left(\alpha_{1}\right)$ is defined by (11).

Theorem 1. Let $f=h+\bar{g}$ be such that $h(z)$ and $g(z)$ are given by (2). Furthermore, let

$$
\begin{aligned}
\sum_{n=1+p}^{\infty}( & n-p \gamma) \Omega \sigma_{n, p}\left(\alpha_{1}\right)\left|a_{n}\right| \\
& +\sum_{n=p}^{\infty}(n+p \gamma) \Omega \sigma_{n, p}\left(\alpha_{1}\right)\left|b_{n}\right| \leq p(1-\gamma) .
\end{aligned}
$$

Then $f(z)$ is orientation preserving in $U$ and $f(z) \in H_{p}\left(\left[\alpha_{1}\right.\right.$, $\left.\left.A_{1}, B_{1}\right], q, s ; \gamma\right)$. 
Proof. The inequality $\left|h^{\prime}(z)\right| \geq\left|g^{\prime}(z)\right|$ is enough to show that $f(z)$ is orientation preserving. Note that

$$
\begin{aligned}
\left|h^{\prime}(z)\right| & \geq p|z|^{p-1}-\sum_{n=1+p}^{\infty} n\left|a_{n}\right||z|^{n-1} \\
& >p-\sum_{n=1+p}^{\infty} n\left|a_{n}\right| \geq p-\sum_{n=1+p}^{\infty} \frac{(n-p \gamma) \Omega \sigma_{n, p}\left(\alpha_{1}\right)}{p(1-\gamma)}\left|a_{n}\right| \\
& \geq \sum_{n=p}^{\infty} \frac{(n+p \gamma) \Omega \sigma_{n, p}\left(\alpha_{1}\right)}{p(1-\gamma)}\left|b_{n}\right| \geq \sum_{n=p}^{\infty} n\left|b_{n}\right| \\
& >\sum_{n=p}^{\infty} n\left|b_{n}\right||z|^{n-1} \geq\left|g^{\prime}(z)\right| .
\end{aligned}
$$

Now we will show that $f(z) \in H_{p}\left(\left[\alpha_{1}, A_{1}, B_{1}\right], q, s ; \gamma\right)$. We only need to show that if (17) holds, then condition (15) is satisfied. Using the fact that $\operatorname{Re}\{w\} \geq p \gamma$ if and only if $\mid p(1-$ $\gamma)+w|\geq| p(1+\gamma)-w \mid$, it suffices to show that

$$
|A(z)+p(1-\gamma) B(z)|-|A(z)-p(1+\gamma) B(z)| \geq 0,
$$

where

$$
\begin{gathered}
A(z)=z \frac{z\left(\theta_{p, q, s}\left[\alpha_{1}, A_{1}, B_{1}\right] h(z)\right)^{\prime}}{-\overline{z\left(\theta_{p, q, s}\left[\alpha_{1}, A_{1}, B_{1}\right] g(z)\right)^{\prime}},} \\
B(z)=\theta_{p, q, s}\left[\alpha_{1}, A_{1}, B_{1}\right] h(z)+\overline{\theta_{p, q, s}\left[\alpha_{1}, A_{1}, B_{1}\right] g(z) .}
\end{gathered}
$$

Substituting for $A(z)$ and $B(z)$ in (17) yields

$$
\begin{aligned}
& |A(z)+p(1-\gamma) B(z)|-|A(z)-p(1+\gamma) B(z)| \\
& =\mid(2 p-p \gamma) z^{p}-\sum_{n=1+p}^{\infty}[n+p(1-\gamma)] \Omega \sigma_{n, p}\left(\alpha_{1}\right) a_{n} z^{n} \\
& \quad-\sum_{n=p}^{\infty}[n-p(1-\gamma)] \Omega \sigma_{n, p}\left(\alpha_{1}\right) b_{n} z^{n} \mid \\
& \quad-\mid-p \gamma z^{p}+\sum_{n=1+p}^{\infty}[n-p(1+\gamma)] \Omega \sigma_{n, p} a_{n} z^{n} \\
& \quad-\sum_{n=p}^{\infty}[n+p(1+\gamma)] \Omega \sigma_{n, p}\left(\alpha_{1}\right) b_{n} z^{n} \mid \\
& \geq(2 p-p \gamma)|z|^{p}-\sum_{n=1+p}^{\infty}[n+p(1-\gamma)] \Omega \sigma_{n, p}\left(\alpha_{1}\right)\left|a_{n}\right||z|^{n} \\
& \quad-\sum_{n=p}^{\infty}[n+p(\gamma-1)] \Omega \sigma_{n, p}\left(\alpha_{1}\right)\left|b_{n}\right||z|^{n}-p \gamma|z|^{p}
\end{aligned}
$$

$$
\begin{gathered}
-\sum_{n=1+p}^{\infty}[n-p(1+\gamma)] \Omega \sigma_{n, p}\left(\alpha_{1}\right)\left|a_{n}\right||z|^{n} \\
-\sum_{n=p}^{\infty}[n+p(1+\gamma)] \Omega \sigma_{n, p}\left(\alpha_{1}\right)\left|b_{n}\right||z|^{n} \\
=2 p(1-\gamma)|z|^{p}-2 \sum_{n=1+p}^{\infty}(n-p \gamma) \Omega \sigma_{n, p}\left(\alpha_{1}\right)\left|a_{n}\right||z|^{n} \\
-2 \sum_{n=p}^{\infty}(n+p \gamma) \Omega \sigma_{n, p}\left(\alpha_{1}\right)\left|b_{n}\right||z|^{n} \\
=2 p(1-\gamma)|z|^{p}\left(1-\sum_{n=1+p}^{\infty} \frac{(n-p \gamma) \Omega \sigma_{n, p}\left(\alpha_{1}\right)}{p(1-\gamma)}\left|a_{n}\right||z|^{n-p}\right. \\
\left.-\sum_{n=p}^{\infty} \frac{(n+p \gamma) \Omega \sigma_{n, p}\left(\alpha_{1}\right)}{p(1-\gamma)}\left|b_{n}\right||z|^{n-p}\right) \\
\geq 2 p(1-\gamma)|z|^{p}\left\{1-\left(\sum_{n=1+p}^{\infty} \frac{(n-p \gamma) \Omega \sigma_{n, p}\left(\alpha_{1}\right)}{p(1-\gamma)}\left|a_{n}\right|\right.\right. \\
\left.\left.+\sum_{n=p}^{\infty} \frac{(n+p \gamma) \Omega \sigma_{n, p}\left(\alpha_{1}\right)}{p(1-\gamma)}\left|b_{n}\right|\right)\right\} .
\end{gathered}
$$

The last expression is nonnegative by (17). This completes the proof of Theorem 1. The harmonic $p$-valent function

$$
\begin{aligned}
f(z)= & z^{p}+\sum_{n=1+p}^{\infty} \frac{p(1-\gamma)}{(n-p \gamma) \Omega \sigma_{n, p}\left(\alpha_{1}\right)} X_{n} z^{n} \\
& +\sum_{n=p}^{\infty} \frac{p(1-\gamma)}{(n+p \gamma) \Omega \sigma_{n, p}\left(\alpha_{1}\right)} \overline{Y_{n} z^{n}}
\end{aligned}
$$

where $\sum_{n=1+p}^{\infty}\left|X_{n}\right|+\sum_{n=p}^{\infty}\left|Y_{n}\right|=1$, shows that the coefficient bound given by (17) is sharp. It is worthy to note that the function of the form (22) belongs to the class $H_{p}\left(\left[\alpha_{1}\right.\right.$, $\left.\left.A_{1}, B_{1}\right], q, s ; \gamma\right)$ for all $\sum_{n=2}^{\infty}\left|X_{n}\right|+\sum_{n=1}^{\infty}\left|Y_{n}\right| \leq 1$ because coefficient inequality (17) holds.

Theorem 2. A function $f(z)$ is in the class $H_{p}\left(\left[\alpha_{1}, A_{1}\right.\right.$, $\left.\left.B_{1}\right], q, s ; \gamma\right)$, if and only if

$$
\begin{aligned}
& \theta_{p, q, s}\left[\alpha_{1}, A_{1}, B_{1}\right] h(z) \\
& *\left[\frac{2 p(1-\gamma) z^{p}+(\zeta-2 p+2 p \gamma+1) z^{p+1}}{(1-z)^{2}}\right] \\
& \left.-\overline{\theta_{p, q, s}\left[\alpha_{1}, A_{1}, B_{1}\right] g(z)^{\prime}}\right] \overline{z^{p}+(\zeta-2 p \zeta-2 p \gamma+1) \overline{z^{p+1}}} \\
& \quad *\left[\frac{2 p(\zeta+\gamma) \bar{z})^{2}}{\left(1-\overline{z^{2}}\right.}\right] \neq 0, \\
& |\zeta|=1 .
\end{aligned}
$$


Proof. From (15), we have the necessary and sufficient condition for $f(z) \in H_{p}\left(\left[\alpha_{1}, A_{1}, B_{1}\right], q, s ; \gamma\right)$ that is,

$$
\begin{aligned}
& \operatorname{Re}\left\{\frac{1}{p(1-\gamma)}\right. \\
& \times\left[\left(z\left(\theta_{p, q, s}\left[\alpha_{1}, A_{1}, B_{1}\right] h(z)\right)^{\prime}\right.\right. \\
& \left.\overline{-z\left(\theta_{p, q, s}\left[\alpha_{1}, A_{1}, B_{1}\right] g(z)\right)^{\prime}}\right) \\
& \times\left(\theta_{p, q, s}\left[\alpha_{1}, A_{1}, B_{1}\right] h(z)\right. \\
& \left.\left.\left.+\overline{\theta_{p, q, s}\left[\alpha_{1}, A_{1}, B_{1}\right] g(z)}\right)^{-1}-p \gamma\right]\right\} \geq 0 .
\end{aligned}
$$

Hence we have the equivalent condition

$$
\begin{aligned}
& \frac{1}{p(1-\gamma)}\left[\left(z\left(\theta_{p, q, s}\left[\alpha_{1}, A_{1}, B_{1}\right] h(z)\right)^{\prime}\right.\right. \\
&\left.-z \overline{\left(\theta_{p, q, s}\left[\alpha_{1}, A_{1}, B_{1}\right] g(z)\right)^{\prime}}\right) \\
& \times\left(\theta_{p, q, s}\left[\alpha_{1}, A_{1}, B_{1}\right] h(z)\right. \\
&\left.\left.+\overline{\theta_{p, q, s}\left[\alpha_{1}, A_{1}, B_{1}\right] g(z)}\right)^{-1}-p \gamma\right] \neq \frac{\zeta-1}{\zeta+1},
\end{aligned}
$$

$|\zeta|=1, \zeta \neq-1,0<|z|<1$. Simple algebraic manipulation of (25) yields

$$
\begin{aligned}
& 0 \neq\left[\left(z\left(\theta_{p, q, s}\left[\alpha_{1}, A_{1}, B_{1}\right] h(z)\right)^{\prime}\right.\right. \\
& \left.-\overline{z\left(\theta_{p, q, s}\left[\alpha_{1}, A_{1}, B_{1}\right] g(z)\right)^{\prime}}\right) \\
& \times\left(\theta_{p, q, s}\left[\alpha_{1}, A_{1}, B_{1}\right] h(z)\right. \\
& \left.\left.+\overline{\theta_{p, q, s}\left[\alpha_{1}, A_{1}, B_{1}\right] g(z)}\right)^{-1}-p \gamma\right] \\
& -p(1-\gamma)\left(\frac{\zeta-1}{\zeta+1}\right) \\
& =\theta_{p, q, s}\left[\alpha_{1}, A_{1}, B_{1}\right] h(z) \\
& *\left[\frac{2 p(1-\gamma) z^{p}+(\zeta-2 p+2 p \gamma+1) z^{p+1}}{(1-z)^{2}}\right] \\
& -\overline{\theta_{p, q, s}\left[\alpha_{1}, A_{1}, B_{1}\right] g(z)} \\
& *\left[\frac{2 p(\zeta+\gamma) \overline{z^{p}}+(\zeta-2 p \zeta-2 p \gamma+1) \overline{z^{p+1}}}{(1-\bar{z})^{2}}\right] .
\end{aligned}
$$

This completes the proof of Theorem 2.
Theorem 3. A function $f(z)=h+\bar{g}$, where $h(z)$ and $g(z)$ are given by (16), is in the class $\overline{H_{p}}\left(\left[\alpha_{1}, A_{1}, B_{1}\right], q, s ; \gamma\right)$, if and only if

$$
\begin{aligned}
& \sum_{n=1+p}^{\infty}(n-p \gamma) \Omega \sigma_{n, p}\left(\alpha_{1}\right)\left|a_{n}\right| \\
& \quad+\sum_{n=p}^{\infty}(n+p \gamma) \Omega \sigma_{n, p}\left(\alpha_{1}\right)\left|b_{n}\right| \leq p(1-\gamma) .
\end{aligned}
$$

Proof. Since $\overline{H_{p}}\left(\left[\alpha_{1}, A_{1}, B_{1}\right], q, s ; \gamma\right) \subset H_{p}\left(\left[\alpha_{1}, A_{1}, B_{1}\right], q\right.$, $s ; \gamma)$, we only need to prove the "only if" part of this theorem. To this end, for functions $f(z)=h+\bar{g}$, where $h(z)$ and $g(z)$ given by (16), we notice that condition

$$
\begin{aligned}
\operatorname{Re}\{( & z\left(\theta_{p, q, s}\left[\alpha_{1}, A_{1}, B_{1}\right] h(z)\right)^{\prime} \\
& \left.-\overline{z\left(\theta_{p, q, s}\left[\alpha_{1}, A_{1}, B_{1}\right] g(z)\right)^{\prime}}\right) \\
& \times\left(\theta_{p, q, s}\left[\alpha_{1}, A_{1}, B_{1}\right] h(z)\right. \\
& \left.\left.+\overline{\theta_{p, q, s}\left[\alpha_{1}, A_{1}, B_{1}\right] g(z)}\right)^{-1}\right\} \geq p \gamma
\end{aligned}
$$

is equivalent to

$$
\begin{gathered}
\operatorname{Re}\left\{\left(p(1-\gamma) z^{p}-\sum_{n=1+p}^{\infty}(n-p \gamma) \Omega \sigma_{n, p}\left(\alpha_{1}\right) a_{n} z^{n}\right.\right. \\
\left.\quad-\sum_{n=p}^{\infty}(n+p \gamma) \Omega \sigma_{n, p}\left(\alpha_{1}\right) \overline{b_{n} z^{n}}\right) \\
\times\left(z^{p}-\sum_{n=1+p}^{\infty} \Omega \sigma_{n, p}\left(\alpha_{1}\right) a_{n} z^{n}\right. \\
\left.\left.-\sum_{n=p}^{\infty} \Omega \sigma_{n, p}\left(\alpha_{1}\right) \overline{b_{n} z^{n}}\right)^{-1}\right\} \geq 0 .
\end{gathered}
$$

The above condition must hold for all $z,|z|=r<1$. Choosing the values of $z$ on the positive real axis where $0 \leq r<1$, we must have

$$
\begin{gathered}
\left(p(1-\gamma)-\sum_{n=1+p}^{\infty}(n-p \gamma) \Omega \sigma_{n, p}\left(\alpha_{1}\right) a_{n} r^{n-1}\right. \\
\left.-\sum_{n=p}^{\infty}(n+p \gamma) \Omega \sigma_{n, p}\left(\alpha_{1}\right) b_{n} r^{n-1}\right) \\
\times\left(1-\sum_{n=1+p}^{\infty} \Omega \sigma_{n, p}\left(\alpha_{1}\right) a_{n} r^{n-1}\right. \\
\left.-\sum_{n=p}^{\infty} \Omega \sigma_{n, p}\left(\alpha_{1}\right) b_{n} r^{n-1}\right)^{-1} \geq 0 .
\end{gathered}
$$

If condition (27) does not hold, then the numerator in (30) is negative for $r$ sufficiently close to 1 . Hence there 
exist $z_{0}=r_{0}$ in $(0,1)$ for which the quotient in $(30)$ is negative. This contradicts the required condition for $f(z) \in \overline{H_{p}}\left(\left[\alpha_{1}, A_{1}, B_{1}\right], q, s ; \gamma\right)$. This completes the proof of Theorem 3 .

\section{Distortion Theorem}

Theorem 4. Let the function $f(z)=h+\bar{g}$, where $h(z)$ and $g(z)$ are given by (16), belong to the class $\overline{H_{p}}\left(\left[\alpha_{1}, A_{1}, B_{1}\right], q, s ; \gamma\right)$. Then for $|z|=r<1$, we have

$$
\begin{aligned}
|f(z)| \leq & \left(1+\left|b_{p}\right|\right) r^{p}+\frac{1}{\Omega \sigma_{1+p, p}\left(\alpha_{1}\right)} \\
& \times\left(\frac{p(1-\gamma)}{[1+p(1-\gamma)]}-\frac{p(1+\gamma)}{[1+p(1-\gamma)]}\left|b_{p}\right|\right) r^{1+p},
\end{aligned}
$$

$$
\begin{aligned}
|f(z)| \geq & \left(1-\left|b_{p}\right|\right) r^{p}-\frac{1}{\Omega \sigma_{1+p, p}\left(\alpha_{1}\right)} \\
& \times\left(\frac{p(1-\gamma)}{[1+p(1-\gamma)]}-\frac{p(1+\gamma)}{[1+p(1-\gamma)]}\left|b_{p}\right|\right) r^{1+p}
\end{aligned}
$$

for $\left|b_{p}\right| \leq(1-\gamma) /(1+\gamma)$. The results are sharp with equality for the functions $f(z)$ defined by

$$
\begin{aligned}
f(z)= & \left(1+\left|b_{p}\right|\right) \overline{z^{p}}-\frac{1}{\Omega \sigma_{1+p, p}\left(\alpha_{1}\right)} \\
& \times\left(\frac{p(1-\gamma)}{[1+p(1-\gamma)]}-\frac{p(1+\gamma)}{[1+p(1-\gamma)]}\left|b_{p}\right|\right) \bar{z}^{1+p}, \\
f(z)= & \left(1-\left|b_{p}\right|\right) \overline{z^{p}}-\frac{1}{\Omega \sigma_{1+p, p}\left(\alpha_{1}\right)} \\
& \times\left(\frac{p(1-\gamma)}{[1+p(1-\gamma)]}-\frac{p(1+\gamma)}{[1+p(1-\gamma)]}\left|b_{p}\right|\right) z^{1+p} .
\end{aligned}
$$

Proof. We only prove the first inequality. The proof for the second inequality is similar and will be omitted. Let $f(z) \in$ $\overline{H_{p}}\left(\left[\alpha_{1}, A_{1}, B_{1}\right], q, s ; \gamma\right)$. Taking the absolute value of $f$, we have

$$
\begin{aligned}
|f(z)| & \leq\left(1+\left|b_{p}\right|\right) r^{p}+\sum_{n=1+p}^{\infty}\left(\left|a_{n}\right|+\left|b_{n}\right|\right) r^{n} \\
& \leq\left(1+\left|b_{p}\right|\right) r^{p}+\sum_{n=1+p}^{\infty}\left(\left|a_{n}\right|+\left|b_{n}\right|\right) r^{1+p}
\end{aligned}
$$

$$
\begin{aligned}
= & \left(1+\left|b_{p}\right|\right) r^{p}+\frac{p(1-\gamma)}{[1+p(1-\gamma)] \Omega \sigma_{1+p, p}\left(\alpha_{1}\right)} \\
& \times \sum_{n=1+p}^{\infty}\left(\frac{[1+p(1-\gamma)] \Omega \sigma_{1+p, p}\left(\alpha_{1}\right)}{p(1-\gamma)}\left|a_{n}\right|\right. \\
& \left.+\frac{[1+p(1-\gamma)] \Omega \sigma_{1+p, p}\left(\alpha_{1}\right)}{p(1-\gamma)}\left|b_{n}\right|\right) r^{1+p} \\
\leq & \left(1+\left|b_{p}\right|\right) r^{p}+\frac{p(1-\gamma)}{[1+p(1-\gamma)] \Omega \sigma_{1+p, p}\left(\alpha_{1}\right)} \\
& \times \sum_{n=1+p}^{\infty}\left(\frac{(n-p \gamma) \Omega \sigma_{n, p}\left(\alpha_{1}\right)}{p(1-\gamma)}\left|a_{n}\right|\right. \\
\leq & \left.\left(1+\frac{(n+p \gamma) \Omega \sigma_{n, p}\left(\alpha_{1}\right)}{p(1-\gamma)}\left|b_{n}\right|\right) r^{1+p} \mid\right) r^{p}+\frac{p(1-\gamma)}{[1+p(1-\gamma)] \Omega \sigma_{1+p, p}\left(\alpha_{1}\right)} \\
& \times\left(1-\frac{(1+\gamma)}{(1-\gamma)}\left|b_{p}\right|\right) r^{1+p} \\
= & \left(1+\left|b_{p}\right|\right) r^{p}+\frac{1}{\Omega \sigma_{1+p, p}\left(\alpha_{1}\right)} \\
& \times\left(\frac{p(1-\gamma)}{[1+p(1-\gamma)]}-\frac{p(1+\gamma)}{[1+p(1-\gamma)]}\left|b_{p}\right|\right) r^{1+p} .
\end{aligned}
$$

This completes the proof of Theorem 4 .

Putting $A_{i}=1(i=1,2, \ldots, q)$ and $B_{i}=1(i=$ $1,2, \ldots, s)$ in Theorem 4 , we obtain the following corollary which modifies the result obtained by Omar and Halim [10, Theorem 2.6].

Corollary 5. Let the function $f(z)=h+\bar{g}$, where $h(z)$ and $g(z)$ are given by $(16)$, belong to the class $\overline{H_{p}}\left(\left[\alpha_{1}\right], q, s ; \gamma\right)$. Then, for $|z|=r<1$, we have

$$
\begin{aligned}
|f(z)| \leq & \left(1+\left|b_{p}\right|\right) r^{p}+\frac{1}{\Psi_{1+p, p}\left(\alpha_{1}\right)} \\
& \times\left(\frac{p(1-\gamma)}{[1+p(1-\gamma)]}-\frac{p(1+\gamma)}{[1+p(1-\gamma)]}\left|b_{p}\right|\right) r^{1+p}, \\
|f(z)| \geq & \left(1-\left|b_{p}\right|\right) r^{p}-\frac{1}{\Psi_{1+p, p}\left(\alpha_{1}\right)} \\
& \times\left(\frac{p(1-\gamma)}{[1+p(1-\gamma)]}-\frac{p(1+\gamma)}{[1+p(1-\gamma)]}\left|b_{p}\right|\right) r^{1+p}
\end{aligned}
$$


for $\left|b_{p}\right| \leq(1-\gamma) /(1+\gamma)$. The results are sharp with equality for the functions $f(z)$ defined by

$$
\begin{aligned}
f(z)= & \left(1+\left|b_{p}\right|\right) \overline{z^{p}}-\frac{1}{\Psi_{1+p, p}\left(\alpha_{1}\right)} \\
& \times\left(\frac{p(1-\gamma)}{[1+p(1-\gamma)]}-\frac{p(1+\gamma)}{[1+p(1-\gamma)]}\left|b_{p}\right|\right) \bar{z}^{1+p}, \\
f(z)= & \left(1-\left|b_{p}\right|\right) \overline{z^{p}}-\frac{1}{\Psi_{1+p, p}\left(\alpha_{1}\right)} \\
& \times\left(\frac{p(1-\gamma)}{[1+p(1-\gamma)]}-\frac{p(1+\gamma)}{[1+p(1-\gamma)]}\left|b_{p}\right|\right) z^{1+p},
\end{aligned}
$$

where $\Psi_{1+p, p}\left(\alpha_{1}\right)=\Omega \sigma_{1+p, p}\left(\alpha_{1}\right)$ with $A_{i}=1(i=1,2, \ldots, q)$ and $B_{i}=1(i=1,2, \ldots, s)$.

\section{Extreme Points}

Theorem 6. Let $f(z)=h+\bar{g}$, where $h(z)$ and $g(z)$ are given by (16). Then $f(z) \in \overline{H_{p}}\left(\left[\alpha_{1}, A_{1}, B_{1}\right], q, s ; \gamma\right)$, if and only if

$$
f(z)=\sum_{n=p}^{\infty}\left(\mu_{n} h_{n}(z)+\eta_{n} g_{n}(z)\right)
$$

where $h_{p}(z)=z^{p}$,

$$
\begin{gathered}
h_{n}(z)=z^{p}-\frac{p(1-\gamma)}{(n-p \gamma) \Omega \sigma_{n, p}\left(\alpha_{1}\right)} z^{n} \\
(n=1+p, 2+p, \ldots), \\
g_{n}(z)=z^{p}+\frac{p(1-\gamma)}{(n+p \gamma) \Omega \sigma_{n, p}\left(\alpha_{1}\right)} \overline{z^{n}} \quad(n=p, 1+p, \ldots),
\end{gathered}
$$

$\mu_{n} \geq 0, \eta_{n} \geq 0, \sum_{n=p}^{\infty}\left(\mu_{n}+\eta_{n}\right)=1$. In particular, the extreme points of the class $\overline{H_{p}}\left(\left[\alpha_{1}, A_{1}, B_{1}\right], q, s ; \gamma\right)$ are $\left\{h_{n}\right\}$ and $\left\{g_{n}\right\}$.

Proof. Suppose that

$$
\begin{aligned}
f(z)= & \sum_{n=p}^{\infty}\left(\mu_{n} h_{n}(z)+\eta_{n} g_{n}(z)\right) \\
= & z^{p}-\sum_{n=1+p}^{\infty} \frac{p(1-\gamma)}{(n-p \gamma) \Omega \sigma_{n, p}\left(\alpha_{1}\right)} \mu_{n} z^{n} \\
& +\sum_{n=p}^{\infty} \frac{p(1-\gamma)}{(n+p \gamma) \Omega \sigma_{n, p}\left(\alpha_{1}\right)} \eta_{n} \overline{z^{n}} .
\end{aligned}
$$

Then

$$
\begin{aligned}
\sum_{n=1+p}^{\infty} & \frac{(n-p \gamma) \Omega \sigma_{n, p}\left(\alpha_{1}\right)}{p(1-\gamma)}\left(\frac{p(1-\gamma)}{(n-p \gamma) \Omega \sigma_{n, p}\left(\alpha_{1}\right)} \mu_{n}\right) \\
& +\sum_{n=p}^{\infty} \frac{(n+p \gamma) \Omega \sigma_{n, p}\left(\alpha_{1}\right)}{p(1-\gamma)}\left(\frac{p(1-\gamma)}{(n+p \gamma) \Omega \sigma_{n, p}\left(\alpha_{1}\right)} \eta_{n}\right) \\
= & \sum_{n=1+p}^{\infty} \mu_{n}+\sum_{n=p}^{\infty} \eta_{n}=1-\mu_{p} \leq 1
\end{aligned}
$$

and so $f(z) \in \overline{H_{p}}\left(\left[\alpha_{1}, A_{1}, B_{1}\right], q, s ; \gamma\right)$.

Conversely, if $f(z) \in \overline{H_{p}}\left(\left[\alpha_{1}, A_{1}, B_{1}\right], q, s ; \gamma\right)$, then

$$
\begin{gathered}
\left|a_{n}\right| \leq \frac{p(1-\gamma)}{(n-p \gamma) \Omega \sigma_{n, p}\left(\alpha_{1}\right)} \quad(n \geq 1+p), \\
\left|b_{n}\right| \leq \frac{p(1-\gamma)}{(n+p \gamma) \Omega \sigma_{n, p}\left(\alpha_{1}\right)} \quad(n \geq p) .
\end{gathered}
$$

Set

$$
\begin{gathered}
\mu_{n}=\frac{(n-p \gamma) \Omega \sigma_{n, p}\left(\alpha_{1}\right)}{p(1-\gamma)}\left|a_{n}\right| \quad(n=1+p, 2+p, \ldots), \\
\eta_{n}=\frac{(n+p \gamma) \Omega \sigma_{n, p}\left(\alpha_{1}\right)}{p(1-\gamma)}\left|b_{n}\right| \quad(n=p, 1+p, \ldots) .
\end{gathered}
$$

Since $0 \leq \mu_{n} \leq 1(n=1+p, 2+p, \ldots)$ and $0 \leq \eta_{n} \leq 1(n=$ $p, 1+p, \ldots), \mu_{p}=1-\sum_{n=1+p}^{\infty} \mu_{n}+\sum_{n=p}^{\infty} \eta_{n} \geq 0$, then we can see that $f(z)$ can be expressed in the form (37). This completes the proof of Theorem 6 .

Now we show that the class $\overline{H_{p}}\left(\left[\alpha_{1}, A_{1}, B_{1}\right], q, s ; \gamma\right)$ is closed under convex combinations of its members.

Theorem 7. The class $\overline{H_{p}}\left(\left[\alpha_{1}, A_{1}, B_{1}\right], q, s ; \gamma\right)$ is closed under convex combination.

Proof. For $i=1,2,3, \ldots$, let $f_{i} \in \overline{H_{p}}\left(\left[\alpha_{1}, A_{1}, B_{1}\right], q, s ; \gamma\right)$, where $f_{i}$ is given by

$$
f_{i}=z-\sum_{n=1+p}^{\infty}\left|a_{n_{i}}\right| z^{n}+\sum_{n=p}^{\infty}\left|b_{n_{i}}\right| \overline{z^{n}}
$$

Then by using Theorem 3, we have

$$
\begin{aligned}
\sum_{n=1+p}^{\infty} & \frac{(n-p \gamma) \Omega \sigma_{n, p}\left(\alpha_{1}\right)}{p(1-\gamma)}\left|a_{n_{i}}\right| z^{n} \\
& +\sum_{n=p}^{\infty} \frac{(n+p \gamma) \Omega \sigma_{n, p}\left(\alpha_{1}\right)}{p(1-\gamma)}\left|b_{n_{i}}\right|^{n} \leq 1 .
\end{aligned}
$$


For $\sum_{n=1}^{\infty} t_{i}=1,0 \leq t_{i} \leq 1$, the convex combination of $f_{i}$ may be written as

$$
\begin{aligned}
\sum_{i=1}^{\infty} t_{i} f_{i}(z)= & z^{p}-\sum_{n=1+p}^{\infty}\left(\sum_{i=1}^{\infty} t_{i}\left|a_{n_{i}}\right|\right) z^{n} \\
& +\sum_{n=p}^{\infty}\left(\sum_{i=1}^{\infty} t_{i}\left|b_{n_{i}}\right|\right) \overline{z^{n}} .
\end{aligned}
$$

Then by (45), we have

$$
\begin{aligned}
\sum_{n=1+p}^{\infty} & \frac{(n-p \gamma) \Omega \sigma_{n, p}\left(\alpha_{1}\right)}{p(1-\gamma)}\left(\sum_{i=1}^{\infty} t_{i}\left|a_{n_{i}}\right|\right) \\
& +\sum_{n=p}^{\infty} \frac{(n+p \gamma) \Omega \sigma_{n, p}\left(\alpha_{1}\right)}{p(1-\gamma)}\left(\sum_{i=1}^{\infty} t_{i}\left|b_{n_{i}}\right|\right) \\
= & \sum_{i=1}^{\infty} t_{i}\left(\sum_{n=1+p}^{\infty} \frac{(n-p \gamma) \Omega \sigma_{n, p}\left(\alpha_{1}\right)}{p(1-\gamma)}\left|a_{n_{i}}\right|\right. \\
& \left.+\sum_{n=p}^{\infty} \frac{(n+p \gamma) \Omega \sigma_{n, p}\left(\alpha_{1}\right)}{p(1-\gamma)}\left|b_{n_{i}}\right|\right) \\
\leq \sum_{i=1}^{\infty} t_{i} & =1 .
\end{aligned}
$$

This is the required condition and so $\sum_{i=1}^{\infty} t_{i} f_{i}(z) \in \overline{H_{p}}\left(\left[\alpha_{1}\right.\right.$, $\left.\left.A_{1}, B_{1}\right], q, s ; \gamma\right)$. This completes the proof of Theorem 7 .

\section{Acknowledgment}

The authors would like to thank the referees of the paper for their helpful suggestions.

\section{References}

[1] J. Clunie and T. Sheil-Small, "Harmonic univalent functions," Annales Academiae Scientiarum Fennicae A, vol. 9, pp. 3-25, 1984.

[2] O. P. Ahuja and J. M. Jahangiri, "Multivalent harmonic starlike functions," Annales Universitatis Mariae Curie-Skłodowska, vol. 55, pp. 1-13, 2001.

[3] E. M. Wright, "The asymptotic expansion of the generalized hypergeometric function," Proceedings of the London Mathematical Society, vol. 46, pp. 389-408, 1946.

[4] H. M. Srivastava and P. W. Karlsson, Multiple Gaussian Hypergeometric Series, Ellis Horwood, Chichester, UK, 1985.

[5] J. Dziok and H. M. Srivastava, "Classes of analytic functions associated with the generalized hypergeometric function," Applied Mathematics and Computation, vol. 103, no. 1, pp. 1-13, 1999.

[6] J. Dziok and R. K. Raina, "Families of analytic functions associated with the Wright generalized hypergeometric function," Demonstratio Mathematica, vol. 37, no. 3, pp. 533-542, 2004.

[7] M. K. Aouf and J. Dziok, "Certain class of analytic functions associated with the Wright generalized hypergeometric function," Journal of Mathematics and Applications, vol. 30, pp. 2332, 2008.
[8] M. K. Aouf, A. Shamandy, A. O. Mostafa, and S. M. Madian, "Certain class of $p$-valent functions associated with the Wright generalized hypergeometric function," Demonstratio Mathematica, vol. 43, no. 1, pp. 40-54, 2010.

[9] G. Murugusundaramoorthy and R. K. Raina, "On a subclass of harmonic functions associated with Wright's generalized hypergeometric functions," Hacettepe Journal of Mathematics and Statistics, vol. 38, no. 2, pp. 129-136, 2009.

[10] R. Omar and S. A. Halim, "Multivalent harmonic functions defined by Dziok-Srivastava operator," Bulletin of the Malaysian Mathematical Sciences Society, vol. 35, no. 3, pp. 601-610, 2012.

[11] R. A. Al-Khal, "Goodman-Rønning-type harmonic univalent functions based on Dziok-Srivastava operator," Applied Mathematical Sciences, vol. 5, no. 9-12, pp. 573-584, 2011.

[12] H. A. Al-Kharsani and R. A. Al-Khal, "Univalent harmonic functions," Journal of Inequalities in Pure and Applied Mathematics, vol. 8, no. 2, article 59, pp. 1-8, 2007.

[13] J. M. Jahangiri, "Harmonic functions starlike in the unit disk," Journal of Mathematical Analysis and Applications, vol. 235, no. 2, pp. 470-477, 1999. 


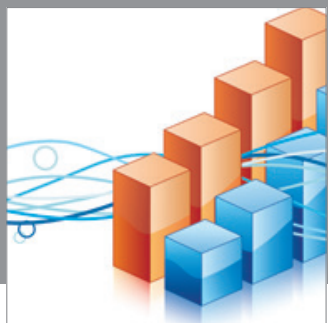

Advances in

Operations Research

mansans

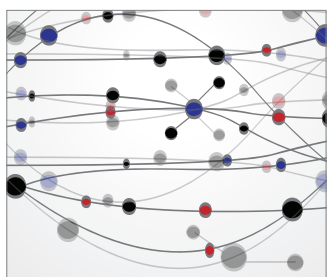

The Scientific World Journal
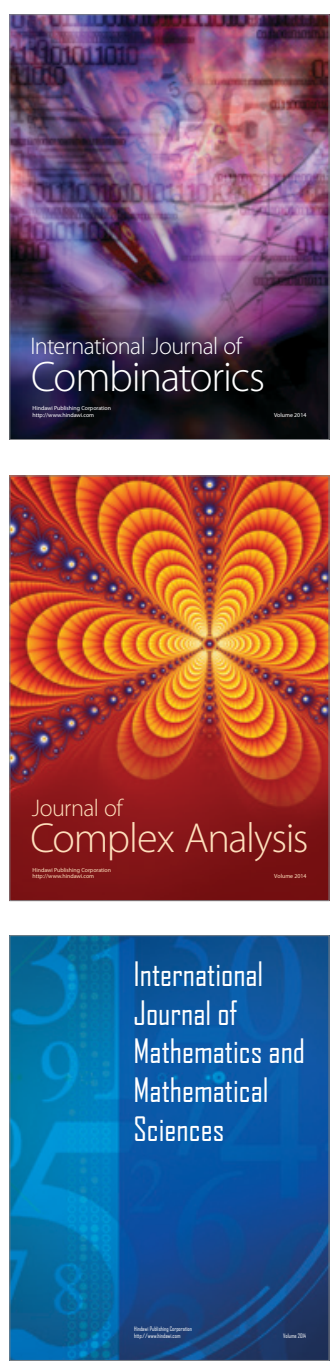
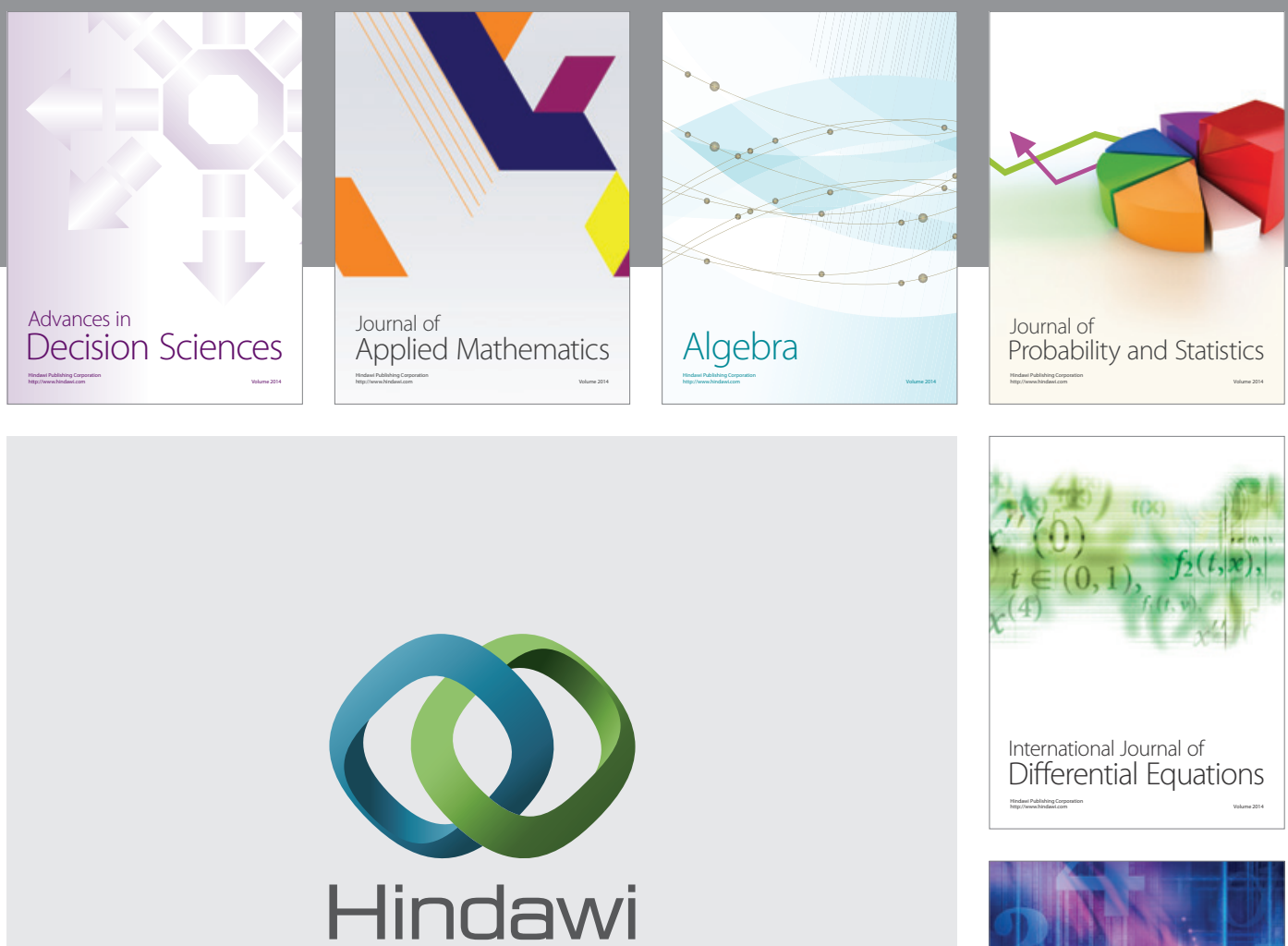

Submit your manuscripts at http://www.hindawi.com
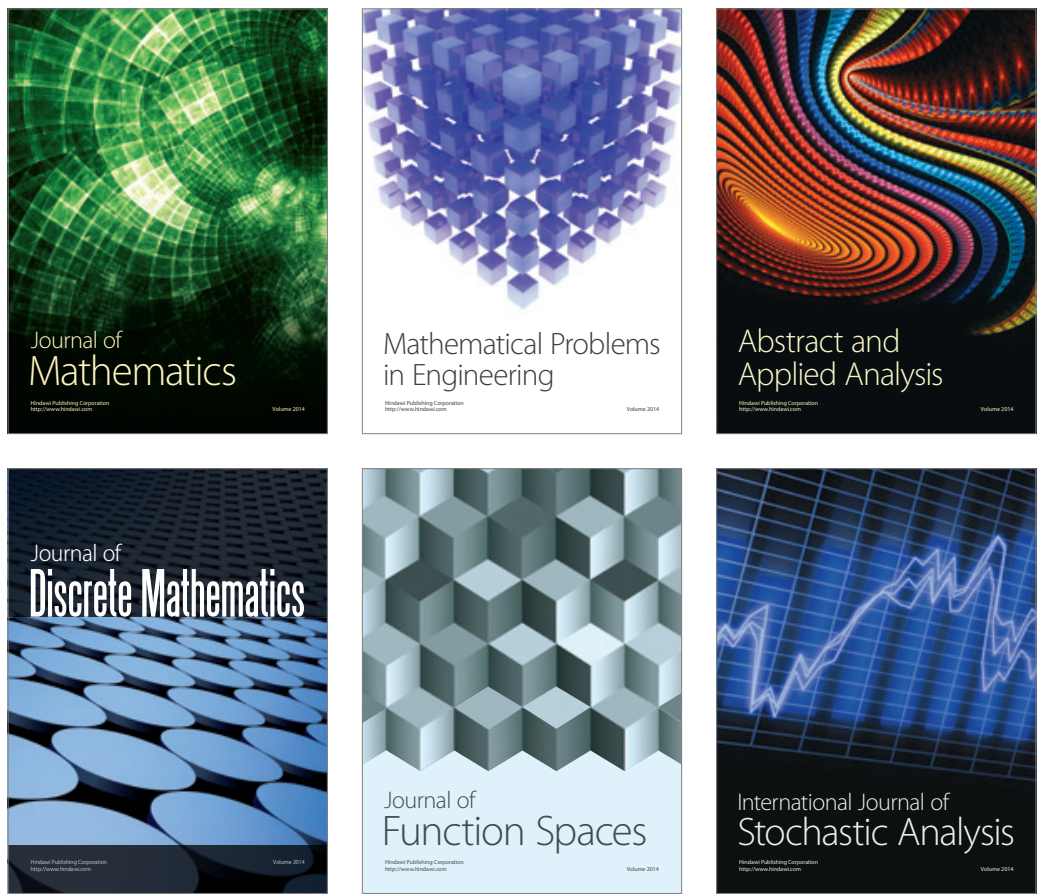

Journal of

Function Spaces

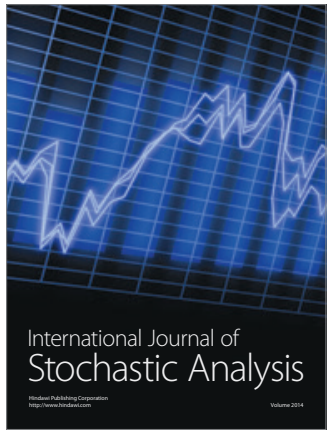

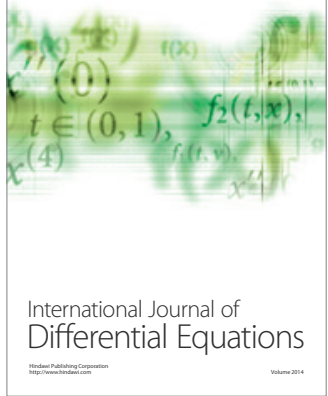
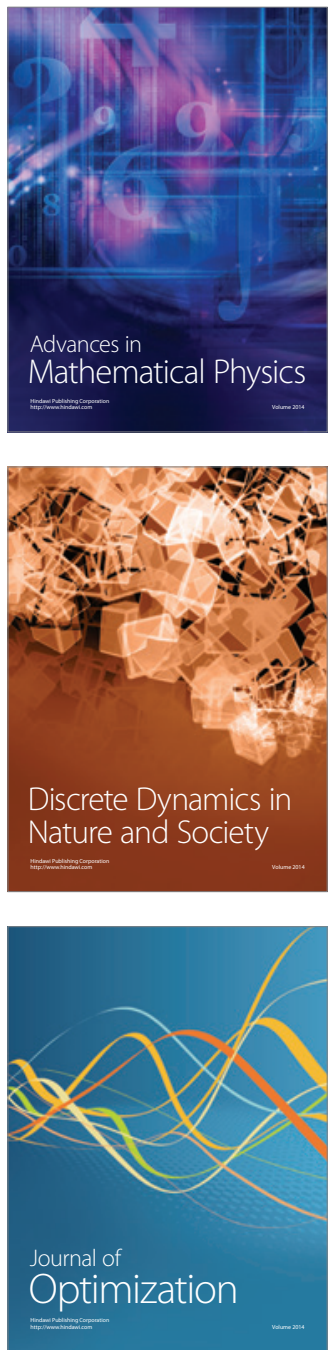